\title{
Poster: Optimizing Tree Reconfiguration to Track Mobile Targets in Sensor Networks.
}

\author{
Wensheng Zhang and Guohong Cao \\ Department of Computer Science \& Engineering \\ The Pennsylvania State University \\ University Park, PA 16802 \\ Email: \{wezhang,gcao\}@cse.psu.edu
}

\begin{abstract}
Most existing work on sensor networks concentrates on finding efficient ways to forward data from the information source to the data centers, and not much work has been done on collecting local data and generating the data report. This paper studies this issue by proposing energy efficient techniques to detect and track a mobile target. We introduce the concept of dynamic convoy tree-based collaboration (DCTC), and formalize it as an optimization problem which needs to find a convoy tree sequence with low energy consumption. We solve the optimization problem in two steps. First, we propose an interception-based reconfiguration algorithm, which can reconfigure the tree structure in an energy efficient way. Second, we develop a theoretical network model, based on which we propose an optimal method for root migration. Extensive experiments are conducted to verify the effectiveness of the proposed method for root migration, and to evaluate and compare the performance of various tree reconfiguration schemes which use different root migration methods and tree reconfiguration algorithms. The results show that the developed optimal method for root migration matches the analytical results, and the tree reconfiguration scheme using the optimal root migration method and the interception-based reconfiguration algorithm has the lowest energy consumption.
\end{abstract}

\section{General Terms}

Algorithm Design

\section{Keywords}

Reconfiguration, Convoy Tree, Target Tracking, Sensor Networks

\section{INTRODUCTION}

*This work was supported in part by the National Science Foundation (CAREER CCR-0092770 and ITR-0219711).

Permission to make digital or hard copies of all or part of this work for personal or classroom use is granted without fee provided that copies are not made or distributed for profit or commercial advantage and that copies bear this notice and the full citation on the first page. To copy otherwise, to republish, to post on servers or to redistribute to lists, requires prior specific permission and/or a fee.

MobiHoc'03, June 1-3, 2003, Annapolis, Maryland, USA.

Copyright 2003 ACM 1-58113-684-6/03/0006 ...\$5.00.
Most existing researches on sensor networks $[1,2]$ concentrate on finding efficient ways to forward data from the information source to the data centers, and not much work has been done on collecting local data and generating the data reports. In this paper, we study this issue by proposing energy efficient techniques to detect and track a mobile target [3], and monitor a particular region surrounding the target in sensor networks. As shown in Figure 1, the sensor nodes surrounding an adversary tank detect and track the tank and its surrounding area which may include enemy soldiers. These nodes collaborate among themselves to aggregate data about the tank as well as its surrounding area, and one of them (i.e., the root) generates a data report. The data report will be forwarded to the data center (the sink), which can be a moving soldier or a static command center. As design goals, the sensor nodes surrounding the moving target should promptly provide robust and reliable tracking information about the mobile target and the region around it in an energy efficient way, and the network should forward this information to the sink in a fast and energy efficient way.

We propose a Dynamic Convoy Tree-based Collaboration $(D C T C)$ framework to detect and track the mobile target. This framework relies on a tree structure called convoy tree ${ }^{1}$, which includes sensor nodes around the moving target, and the tree is dynamically configured to add some nodes and prune some nodes as the target moves. Figure 1 illustrates how to use the convoy tree to track a mobile target. As the target first enters the detection region, sensor nodes that can detect the target collaborate with each other to select a root and construct a convoy tree. Relying on the convoy tree, the root collects information from the sensor nodes and refine these information to obtain more complete and accurate information about the target. As the target moves, the convoy tree reconfigures itself. The sensor nodes in the area where the target is expected to visit in the near future are proactively added to the tree. Some nodes in the tree that become far away from the target are pruned from the tree. As the convoy tree reconfigures itself, the root may also need to be changed to optimize the communication overhead. Figure 1 (b) shows how the convoy tree reconfigures itself with a new root.

Our study focuses on reconfiguring the convoy tree in an

\footnotetext{
${ }^{1}$ We use the word "convoy tree" to differentiate it from the normal tree structure since convoy tree is a moving tree which tracks the target.
} 


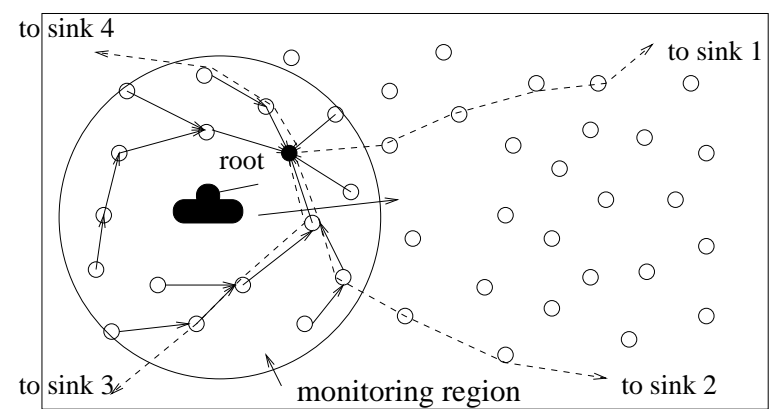

(a)

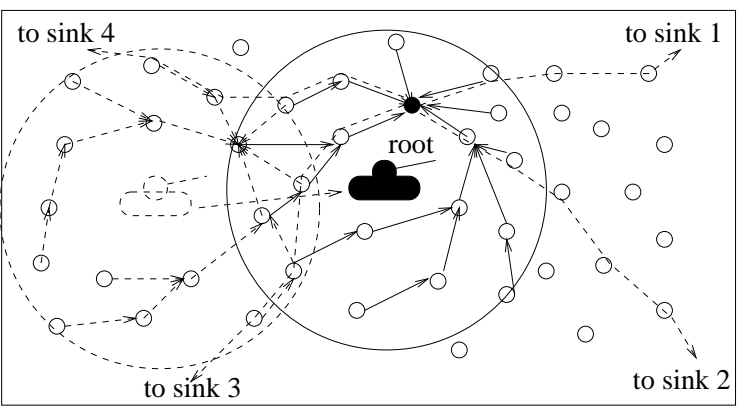

(b)

Figure 1: Using convoy tree to track the target

energy efficient way as the target moves. This problem is first formalized to be an optimization problem of finding a min-cost convoy tree sequence with a high tree coverage. Then, we divide the problem into two subproblems and solve them separately. The first subproblem is how to migrate the root of the tree when the target moves; the second one is how to reconfigure the rest part of the tree after root migration.

\section{ENERGY EFFICIENT TREE RECONFIG- URATION SCHEMES}

We propose two algorithms for tree reconfiguration after root migration. The complete reconfiguration algorithm is designed to minimize the amount of energy consumption in the data collection processes, but it may increase the reconfiguration overhead. In this scheme, the new root needs to broadcast a reconfiguration announcement to its neighbors. All the nodes in the tree, are required to change their locations, after receiving the announcement, to minimize their levels in the new tree and broadcast the changes. The interception-based reconfiguration algorithm is designed to reduce the communication overhead associated with the complete reconfiguration scheme. In this scheme, only the nodes close to the line that is vertical to the line connecting the old root and the new root are required to change their locations, to minimize their levels in the new tree. Since it only reconfigures a small part of the existing tree, the energy consumption is reduced at the cost of some delay increase.

We also develop an optimal method for root migration, which can be used to decide when the root of the convoy tree should be migrated and where the root should be migrated to. In this method, the root keeps monitoring its distance to the target. When the distance becomes larger than a certain threshold (denoted as $d$ ), it will be replaced by the node which is closest to the center of the current monitoring region. The value of $d$ affects the amount of energy consumed in data collection and tree reconfiguration. To find the optimal value of $d$, we develop a model and analyze the impacts of various system parameters on the selection of $d$. This model is based on an ideal sensor network model that nodes are densely and uniformally distributed. When the complete tree reconfiguration scheme or the interception-based tree reconfiguration scheme is used, the major formula for computing the optimal value of $d$ are derived and the numeric results are provided. In spite of the strict assumption, our computation and simulation results have demonstrated that it is very effective when extended to more practical scenarios.

Extensive simulations are conducted to evaluate the effectiveness of the optimal method for root migration, and compare the performance of various tree reconfiguration schemes using various root migration methods. Simulation results show that the tree reconfiguration scheme using the optimal root migration method and the interception-based reconfiguration algorithm has the lowest energy consumption.

\section{CONCLUSIONS}

This paper studied the problem of detecting and tracking a mobile target in an energy efficient way. We introduced the concept of dynamic convoy tree-based collaboration (DCTC), and formalized it as an optimization problem which needs to find a convoy tree sequence with low energy consumption. To solve the optimization problem, we proposed an interception-based reconfiguration algorithm, which can efficiently reconfigure the tree structure, and developed a theoretical network model is developed, based on which an optimal method for root migration has been proposed. Simulation results showed that the developed optimal method for root migration matches the analytical results, and the tree reconfiguration scheme using the optimal root migration method and the interception-based reconfiguration algorithm has the lowest energy consumption.

\section{REFERENCES}

[1] C. Intanagonwiwat, R. Govindan, and D. Estrin, "Directed Diffusion: A Scalable and Robust Communication," MobiCOM '00, August 2000.

[2] F. Ye, H. Luo, J. Cheng, S. Lu, and L. Zhang, "A Two-Tier Data Dissemination Model for Large-scale Wireless Sensor Networks," ACM International Conference on Mobile Computing and Networking (MOBICOM'02), 2002, September 2002.

[3] F. Zhao,J. Shin and J. Reich, "Information-driven Dynamic Sensor Collaboration for Tracking Applications," IEEE Signal Processing Magazine, March 2002. 